\title{
Complete response of MSI-high metastatic colon cancer following treatment with regorafenib: A case report
}

\author{
HYUNGJOO BAIK ${ }^{1}$, HEE JU LEE ${ }^{1}$, JUEUN PARK ${ }^{1}$, HA YOUNG PARK ${ }^{2}$, \\ JINYOUNG PARK ${ }^{3}$, SUNSEONG LEE ${ }^{4}$ and KI BEOM BAE ${ }^{1}$ \\ Departments of ${ }^{1}$ Surgery, ${ }^{2}$ Pathology, ${ }^{3}$ Radiology and ${ }^{4}$ Nuclear Medicine, Inje University, \\ College of Medicine, Busan Paik Hospital, Busan 47392, Republic of Korea
}

Received February 12, 2021; Accepted August 6, 2021

DOI: $10.3892 / \mathrm{mco} .2021 .2405$

\begin{abstract}
Regorafenib has been demonstrated to prolong survival in patients with metastatic colorectal cancer refractory to standard chemotherapy. However, overall survival is limited to 2.5 months. The present report describes a unique case of metastatic colon cancer, which showed a complete response to regorafenib. A 54-year-old woman was diagnosed with right colon cancer obstruction with peritoneal seeding. The patient underwent laparoscopic right hemicolectomy, and the pathology was T4aN2bM1, moderately differentiated adenocarcinoma with high microsatellite instability (MSI-H) and wild-type KRAS/NRAS. The first-line chemotherapy was fluorouracil, leucovorin and irinotecan with cetuximab. After 12 cycles, recurrence at the anastomotic site was identified. The patient underwent palliative colectomy, and superior mesenteric artery (SMA) lymph node metastases were evident. The patient received second-line chemotherapy of fluorouracil, leucovorin and oxaliplatin with bevacizumab. Progression of metastasis to the right common iliac lymph nodes was detected after only four cycles of therapy. Thereafter, the patient received regorafenib as third-line therapy, starting with $160 \mathrm{mg}$ for two cycles and reducing the dose thereafter, for a total of 17 cycles. The previously confirmed SMA lymph node metastasis had disappeared after the seventh cycle, and the right common iliac lymph node metastasis was not visible on CT after the 16th cycle. The patient decided to terminate regorafenib and has not experienced recurrence 2 years since treatment cessation. This is the first report of refractory metastatic colon cancer with MSI-H showing a complete response to regorafenib. Further studies are required to investigate the efficacy of regorafenib in refractory metastatic colon cancer with MSI-H and to elucidate the mechanism of remission.
\end{abstract}

Correspondence to: Dr Ki Beom Bae, Department of Surgery, Inje University, College of Medicine, Busan Paik Hospital, Bokji-ro 75, Busangjin, Busan 47392, Republic of Korea

E-mail: bkbsur@yahoo.co.kr; bkbsur@hanmail.net

Key words: colon cancer, stage IV, complete response, regorafenib, high microsatellite instability

\section{Introduction}

Regorafenib is a novel oral multikinase inhibitor that has shown antitumor activity in various gastrointestinal cancers, including colorectal cancer and gastrointestinal stromal tumor (1). The CORRECT and CONCUR trials have been significant benchmarks in the management of metastatic colorectal cancer (mCRC), demonstrating that regorafenib can slow disease progression and increase the median overall survival (OS) in patients with mCRC $(2,3)$. Those double-blind phase 3 studies showed that the addition of regorafenib to best supportive care prolongs the median OS by up to 2.5 months and progression-free survival (PFS) by up to 1.5 months compared with the addition of placebo in patients with mCRC who had progressed after standard therapy failure. Although disease control [partial response (PR) plus stable disease] was achieved in $51 \%$ of the patients, only $4 \%$ showed a PR, and none showed a complete response (CR). Yoshino et al (4) recently reported a case of mCRC that responded well to regorafenib, resulting in a 2-year-long therapy with a PR. Here, we report the very first case of mCRC that was refractory to conventional chemotherapy such as fluorouracil, leucovorin and irinotecan (FOLFIRI) with cetuximab and fluorouracil, leucovorin and oxaliplatin (FOLFOX) with bevacizumab, but showed a CR to regorafenib. Our patient was a MSI-H, right sided advanced colon cancer patient who had extensive sunlight exposure to her torso. We believe these factors may have contributed to the patient's excellent response to regorafenib, resulting in a prolonged state of $\mathrm{CR}$ even after treatment cessation. The mechanism behind CR should be further elucidated in future studies; for now, we have suggested some possible theories.

\section{Case report}

A 54-year-old woman with abdominal pain and nausea was diagnosed with hepatic flexure colon cancer obstruction with peritoneal seeding in March 2016 (Fig. 1). The patient underwent palliative laparoscopic right hemicolectomy due to obstruction signs, with pathologic report of T4aN2bM1 moderately differentiated adenocarcinoma with lymphovascular invasion and perineural invasion. The tumor also had epidermal growth factor (EGFR) positivity, high microsatellite instability (MSI-H), wild-type KRAS/NRAS, and safety 

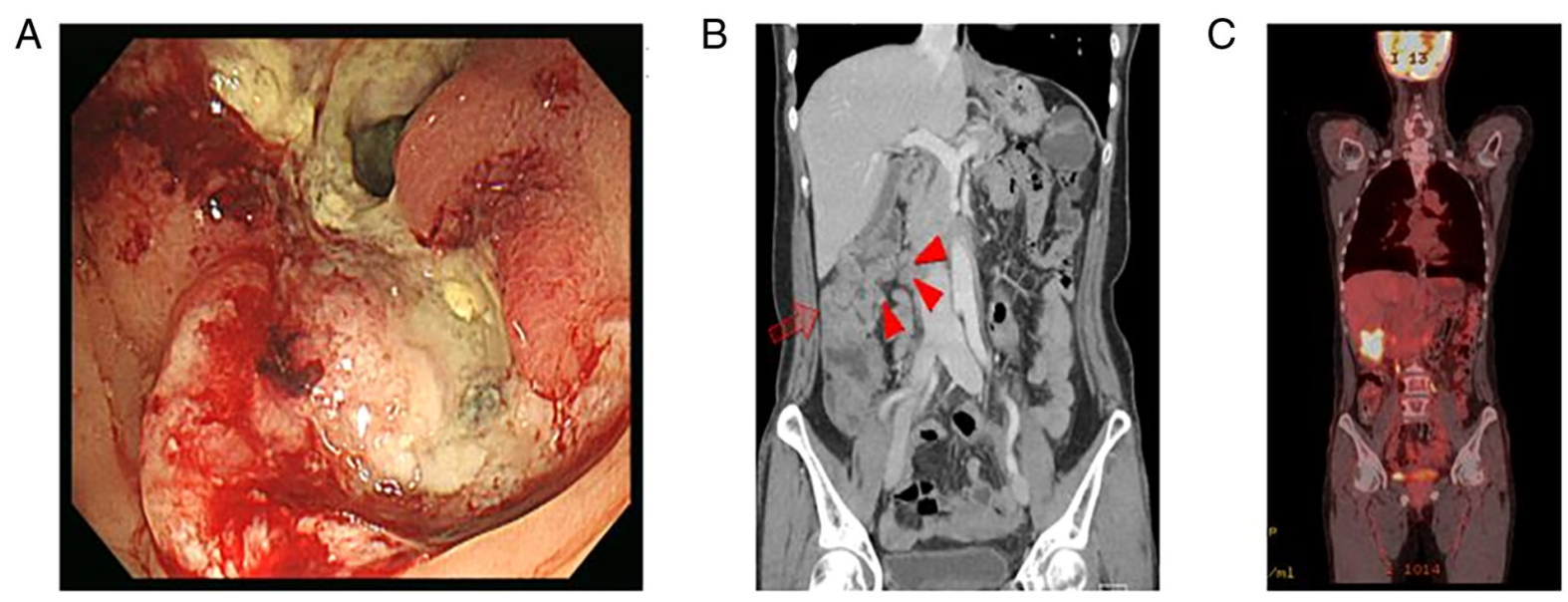

Figure 1. Preoperative images. (A) Colonoscopy findings of the obstructive cancer. (B) Abdominopelvic CT images of ascending colon cancer obstruction (arrow) with pericolic lymph node enlargement (arrowheads). (C) Positron emission tomography-CT images.

A

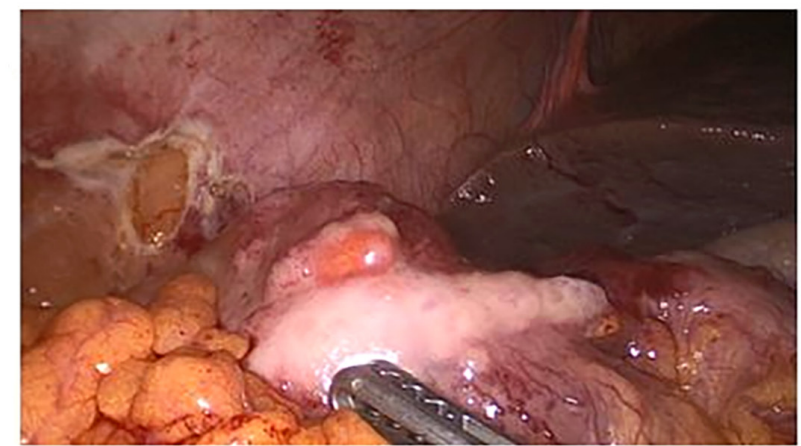

C

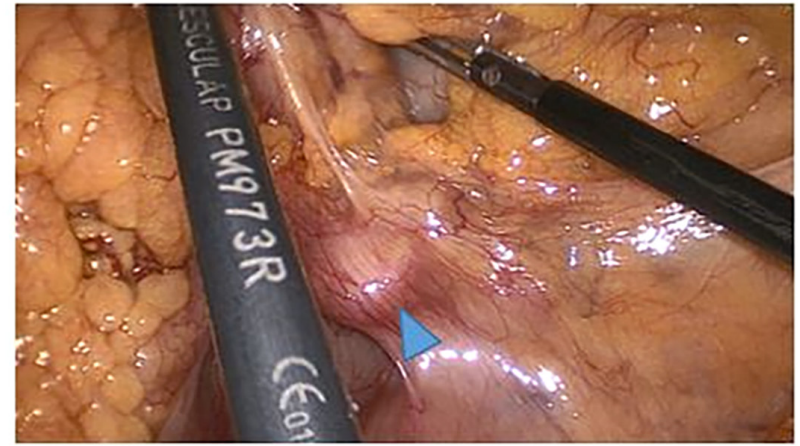

$\mathrm{E}$

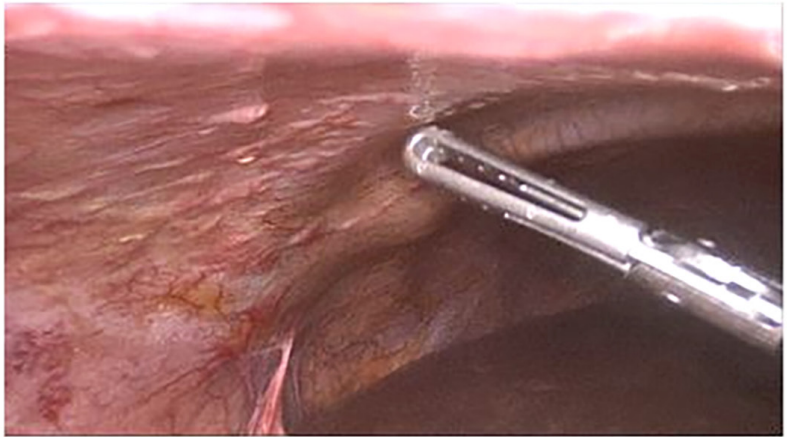

$\mathrm{B}$

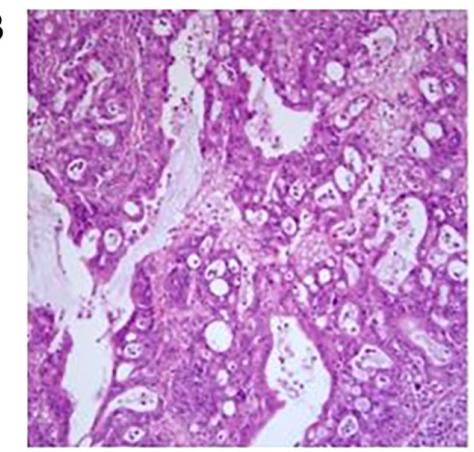

D

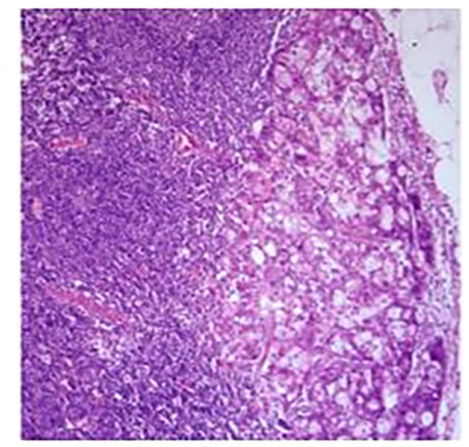

$\mathrm{F}$

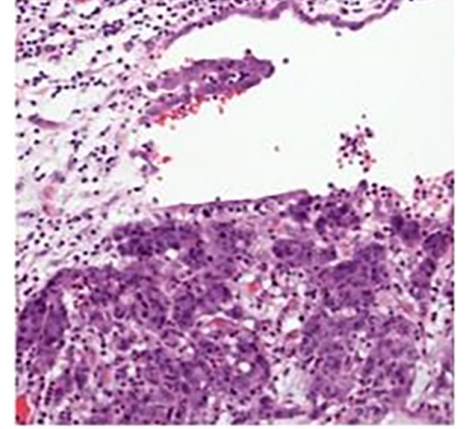

Figure 2. Intraoperative and pathologic findings. (A) Laparoscopic view of the exophytic primary tumor. (B) Pathologic findings of the primary tumor showing fused/cribriform glands and extracellular mucin pools (H\&E staining; magnification, x200). (C) Laparoscopic view of pericolic lymph node enlargement. (D) Pathologic findings of the metastatic tumor in pericolic lymph node (H\&E staining; magnification, x200). (E) Laparoscopic view of peritoneal seeding. (F) Pathologic findings of peritoneal seeding (H\&E staining; magnification, $\mathrm{x} 200$ ).

proximal and distal margins of 7 and $11 \mathrm{~cm}$, respectively. The peritoneal seeding mass was partially removed for biopsy confirmation, which revealed pathologically metastatic adenocarcinoma (Fig. 2). Shortly after palliative laparoscopic right 


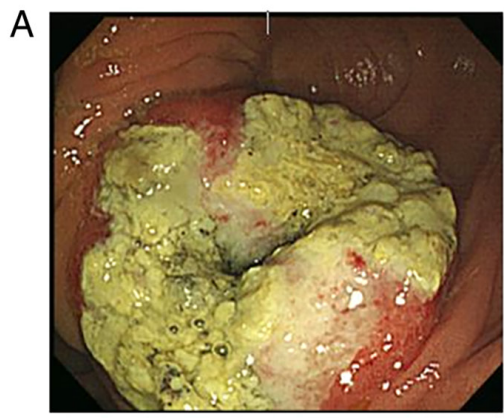

B

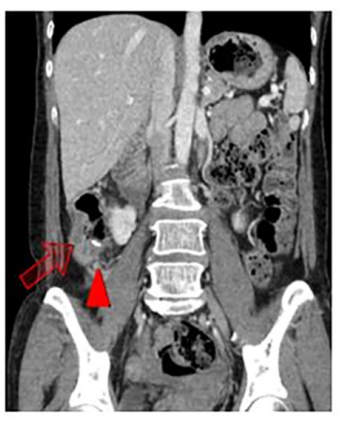

$\mathrm{D}$

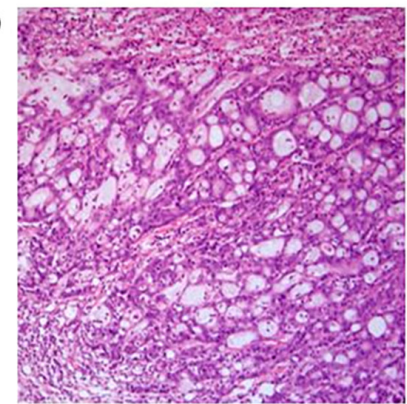

$\mathrm{E}$

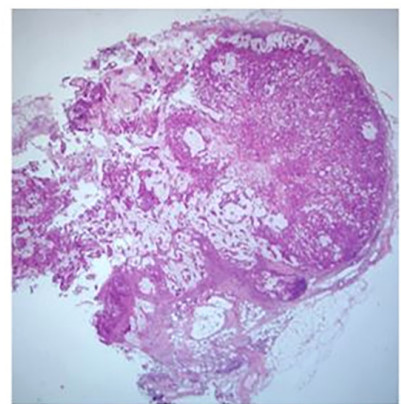

C

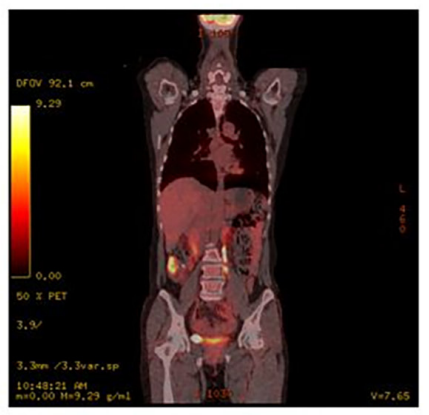

$\mathrm{F}$

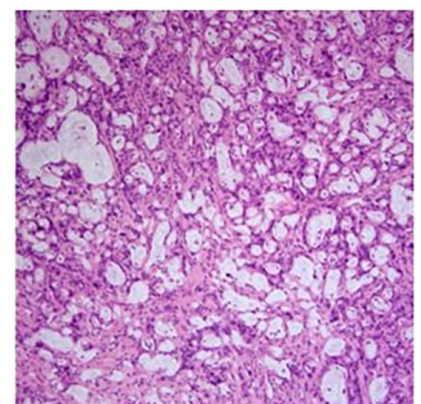

Figure 3. Local recurrence after 12 cycles of fluorouracil, leucovorin and irinotecan plus cetuximab. (A) Recurrence at the anastomosis site confirmed by colonoscopy. (B) Recurrence at the anastomosis site (arrow) with pericolic lymph node enlargement (arrowhead) on abdominopelvic CT images. (C) Positron emission tomography-CT images showing high fluorodeoxyglucose uptake at the anastomosis site. (D) Pathologic evidence of a locally recurrent tumor showing histologic features similar to those of the primary tumor (H\&E staining; magnification, $\mathrm{x} 200$ ). H\&E staining of a metastatic tumor in the superior mesenteric artery lymph node at (E) low power (magnification, x12.5) and (F) high power (magnification, x200).

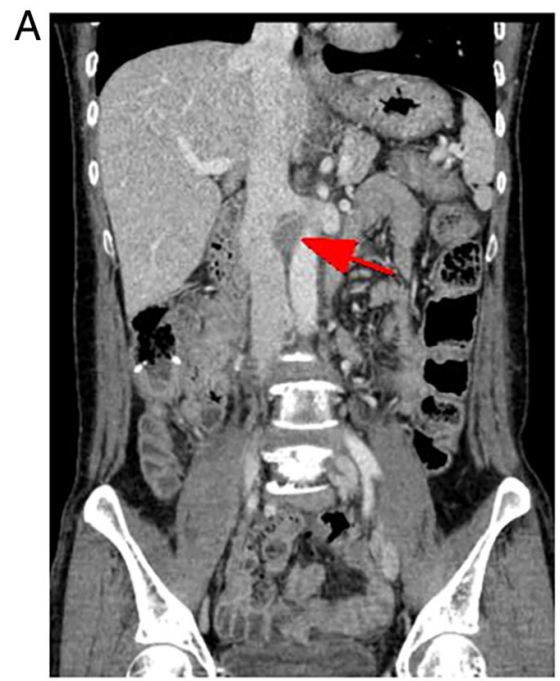

B

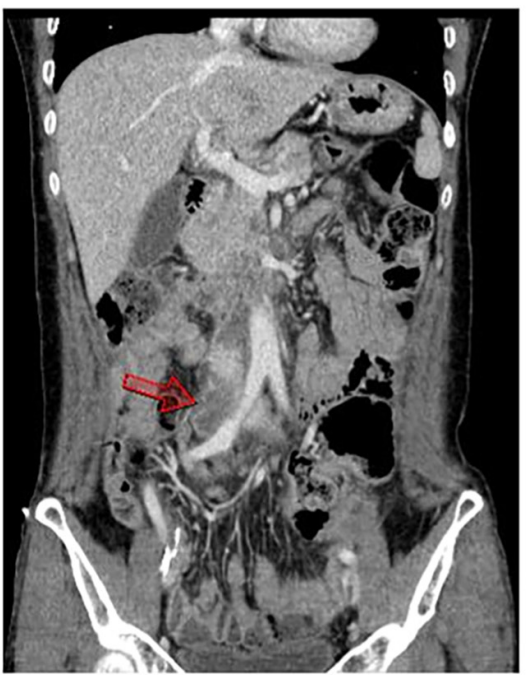

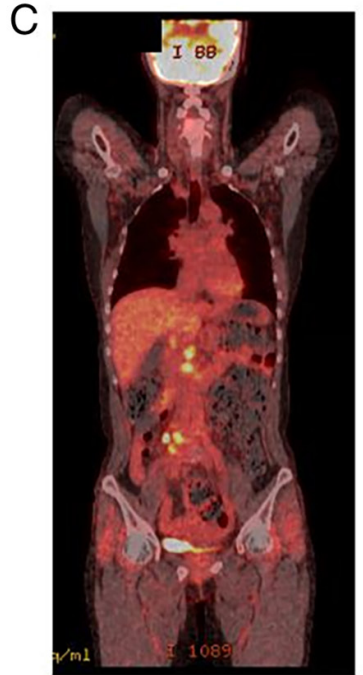

Figure 4. Distant lymph node metastasis after anastomosis site colectomy and lymphadenectomy followed by four cycles of fluorouracil, leucovorin and oxaliplatin plus bevacizumab (pre-regorafenib). (A) APCT showing superior mesenteric artery lymph node metastasis with clip marking (arrow). (B) APCT showing right common iliac lymph node metastasis (arrow). (C) Positron emission tomography-CT showing multifocal lymph node metastasis. APCT, abdominopelvic CT.

hemicolectomy, the patient received 12 cycles of FOLFIRI with cetuximab. After the 12th cycle, local anastomosis site recurrence and pericolic lymph node metastasis were observed (Fig. 3). In October 2016, the patient underwent palliative colectomy with lymph node dissection, which revealed 10/18 positive lymph nodes. During surgery, SMA lymph node metastasis was evident, which was confirmed by selective lymph node retrieval and permanent biopsy. Metal clips were placed in the area of lymph node metastasis for follow-up.
Following recovery, the patient received second-line chemotherapy consisting of FOLFOX with bevacizumab. Progression of metastasis to the right iliac lymph nodes was detected in January 2017 after only four cycles of FOLFOX with bevacizumab (Fig. 4). Thereafter, the patient received regorafenib, starting with $160 \mathrm{mg}$ for two cycles, which was quickly reduced to $120 \mathrm{mg}$ for five cycles according to the Common Terminology Criteria for Adverse Events (CTCAE version 5) indicating grade 3 palmar-plantar erythrodysesthesia 
A

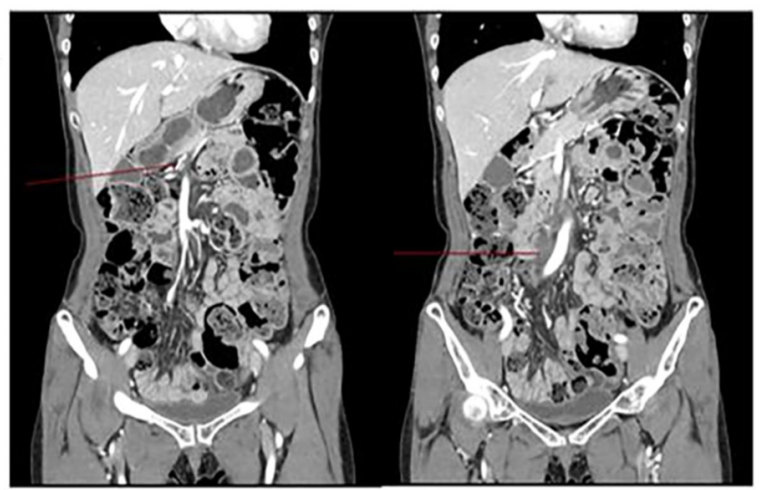

B

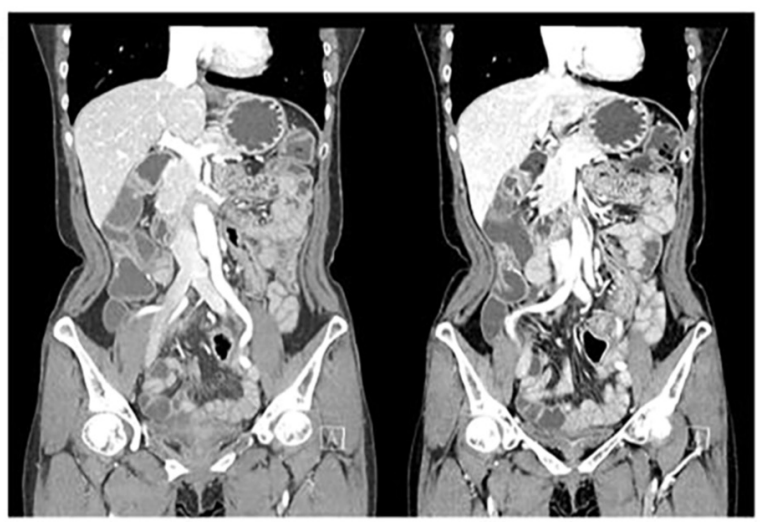

Figure 5. Responses to regorafenib. (A) Disappearance of the superior mesenteric artery lymph node (with clips) but persistence of the right iliac lymph node metastasis after seven cycles of regorafenib. (B) Disappearance of the right iliac lymph node metastasis along with other lymph node metastases after 16 cylces of regorafenib. A complete response was observed.

syndrome (5). Symptoms were partially alleviated after dose reduction, but because grade 2 palmar-plantar erythrodysesthesia syndrome persisted, the patient requested a further dose reduction; therefore, she received $80 \mathrm{mg}$ of regorafenib during cycles 8-12. After the seventh cycle, the previously confirmed SMA lymph node metastasis had disappeared on computed tomography (CT) (Fig. 5A). From cycle 13 to 17, the patient requested a further dose reduction to $40 \mathrm{mg}$ due to general weakness. The right common iliac lymph node metastasis was no longer visible on CT after the cycle 16 (Fig. 5B). The patient decided to terminate chemotherapy and has not experienced recurrence at 2 years since treatment cessation. The clinical effect of regorafenib monotherapy was classified as a CR according to the Response Evaluation Criteria in Solid Tumors (RECIST) v1.1 (6). CT was used for periodic screening, and ${ }^{18} \mathrm{~F}$-fluorodeoxyglucose positron emission tomography (PET)-CT was used to confirm the CR (Fig. 6). The levels of two tumor markers, carcinoembryonic antigen (CEA) and carbohydrate antigen (CA) 19-9, were higher during chemotherapy, but decreased during regorafenib treatment and became within normal range at the time of termination (3.05 ng/ml and $7.35 \mathrm{U} / \mathrm{ml}$, respectively) (Fig. 7). The patient did not suffer from any adverse events aside from palmar-plantar erythrodysesthesia syndrome and mild fatigue. While the patient underwent regorafenib monotherapy, she exposed herself to extensive sunlight for at least $1 \mathrm{~h}$ per day (Fig. 8). The patient's vitamin D $\left(\left(1 \alpha 25-(\mathrm{OH})_{2}\right.\right.$ Vitamin $\left.\mathrm{D}_{3}\right)$ level was not recorded during therapy but was much higher
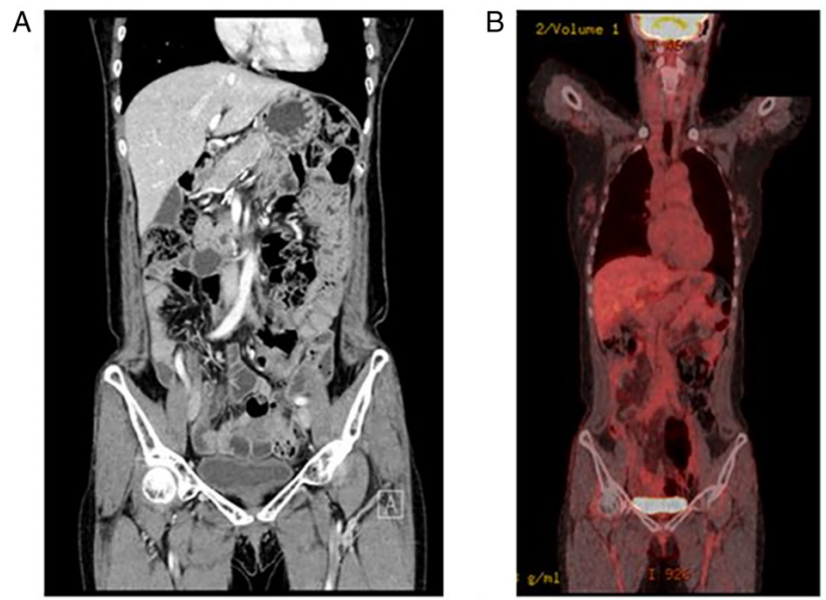

Figure 6 . The complete response persisting at 2 years after cessation of regorafenib treatment. Lack of recurrence was indicated by (A) abdominopelvic $\mathrm{CT}$ and confirmed by (B) positron emission tomography-CT.

$(68.49 \mathrm{pg} / \mathrm{ml})$ than the normal range $(19.6-54.3 \mathrm{pg} / \mathrm{ml})$ after CR achievement, without any supplements.

\section{Discussion}

Prior to the introduction of regorafenib for mCRC treatment, patients who were not responsive to conventional palliative chemotherapy basically had no other treatment option. However, the CORRECT and CONCUR trials showed that both the PFS and OS of $\mathrm{mCRC}$ patients were prolonged following regorafenib treatment, compared with the placebo groups $(\mathrm{P}<0.0001$ and $\mathrm{P}=0.0016)$, with a high disease control rate of up to $51 \%(2,3)$. Unfortunately, no patients in either study showed a CR, and only a few previously reported cases indicated radiological responses $(4,7)$. More recent studies of regorafenib have reported similar results, with no patients showing a CR and only a few showing a PR $(0-3 \%)(8,9)$. Our patient, who initially had MSI-high, right-sided colon cancer with peritoneal carcinomatosis, progressed to SMA and paraaortic lymph node metastases despite conventional chemotherapy. Progression led to cessation of chemotherapy and administration of regorafenib, which resulted in a CR according to both radiological studies and serum tumor marker levels, consistent with the RECIST criteria. Furthermore, the patient has not experienced recurrence at 2 years since cessation of regorafenib. The CEA level was initially normal but increased dramatically due to diffuse paraaortic lymph node metastasis and then approached the normal range soon after the patient started regorafenib, remaining normal even after treatment cessation. However, the CA 19-9 level appeared to be the more sensitive marker in this patient, showing significant elevation at the time of cancer progression. Despite first- and second-line chemotherapy and targeted therapy, the CA 19-9 level continued to increase with cancer progression and only gradually decreased during regorafenib treatment (Fig. 7).

To our knowledge, this is the first case of a CR in a patient with $\mathrm{mCRC}$ treated with regorafenib; only a handful of other gastrointestinal cancer cases have shown a CR following regorafenib treatment. Notably, the patient's cancer not only stopped progressing but completely regressed. We 


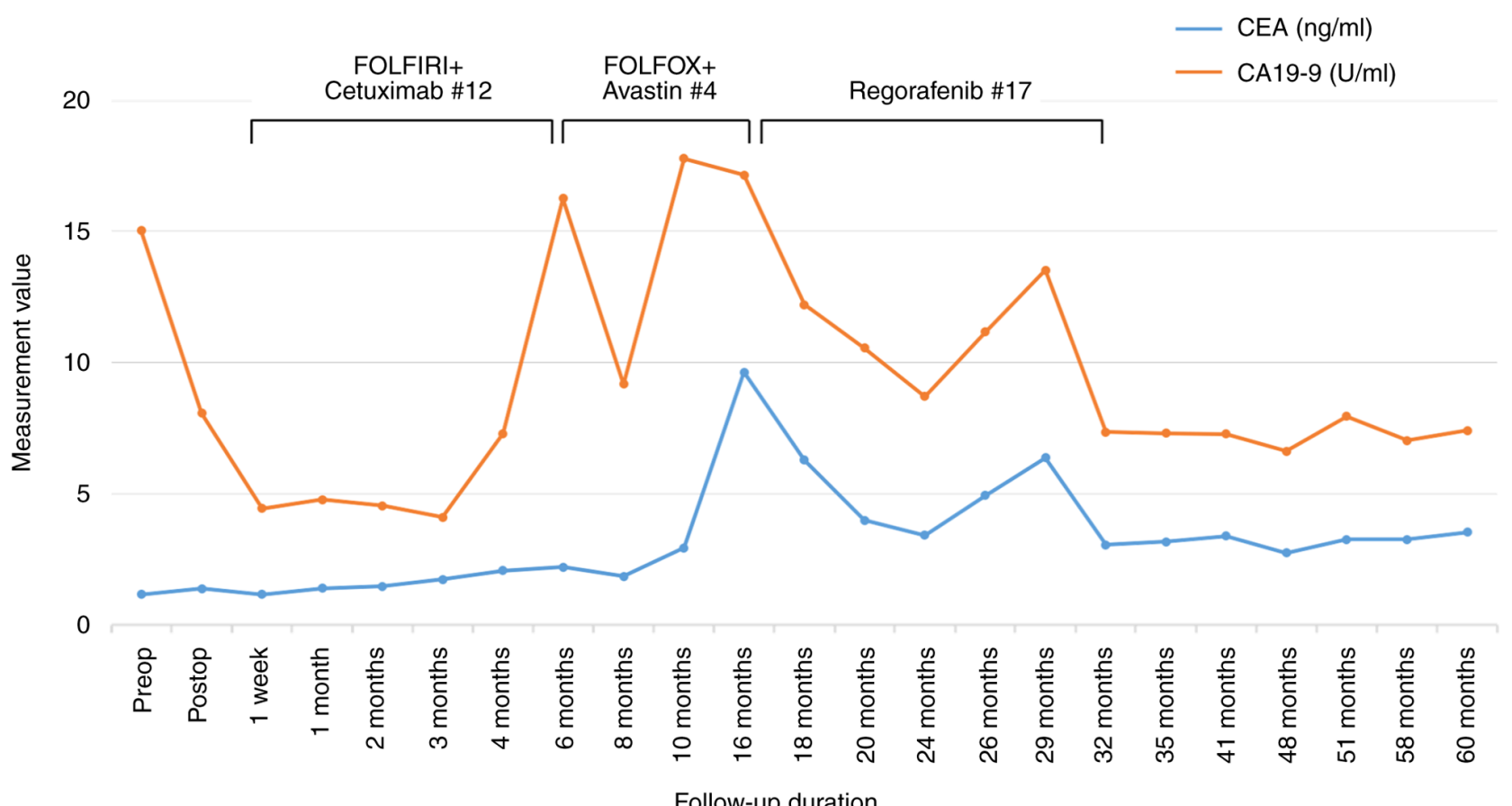

Figure 7. CEA and CA 19-9 levels of the patient throughout the treatment duration. Recurrence was accompanied by elevated CEA or CA 19-9 levels. CA 19-9 appeared to be the more sensitive marker in this patient. FOLFIRI, fluorouracil, leucovorin and irinotecan; FOLFOX, fluorouracil, leucovorin and oxaliplatin; preop, preoperative; postop, postoperative; mo, months.

hypothesize the following reasons for the sudden disappearance of the previously chemotherapy-resistant tumor following regorafenib treatment. MSI-high tumors have a high mutational burden, which increases the likelihood of tumor exposure to the innate immune system $(10,11)$. High MSI is also an effective biomarker of the response to immune-check inhibitor (ICI) in colon cancer, compared with the previously established marker PD-1. Thus, the mutational burden appears to be a more significant factor in immune system escape compared with PD-1; consequently, high MSI is an important surrogate marker $(12,13)$. Increased antitumor immunity is among the pharmacologic traits of regorafenib $(14,15)$, which inhibits CSF1R, a tyrosine kinase receptor involved in macrophage proliferation. CSF1R inhibition by regorafenib may reduce the recruitment of tumor-associated macrophages to the tumor bed, limiting their function, as demonstrated in a model of highly aggressive murine CT26 metastatic colon cancer (16,17). Regorafenib also increases cytotoxic T cells and inhibits the MAPK and JAK1/2-STAT axis, thereby attenuating IFN $\gamma$-induced PD-L1 and IDO1 expression and inducing immune cell attacks (18). The REGONIVO study reported steady tumor regression after treatment with a combination of regorafenib and ICI (19). Based on these studies, increased tumor recognition after regorafenib treatment and decreased tumor immune system escape after ICI treatment appear very promising. Therefore, we suggest that the increased tumor burden due to MSI-H status led to elevated tumor antigenicity, along with enhanced tumor recognition of the immune cells, by regorafenib in our patient, thereby inducing remission. However, this hypothesis should be further evaluated in larger sample sizes.

Another factor that may have influenced our patient's outcome is that she exposed her torso to sunlight for at least

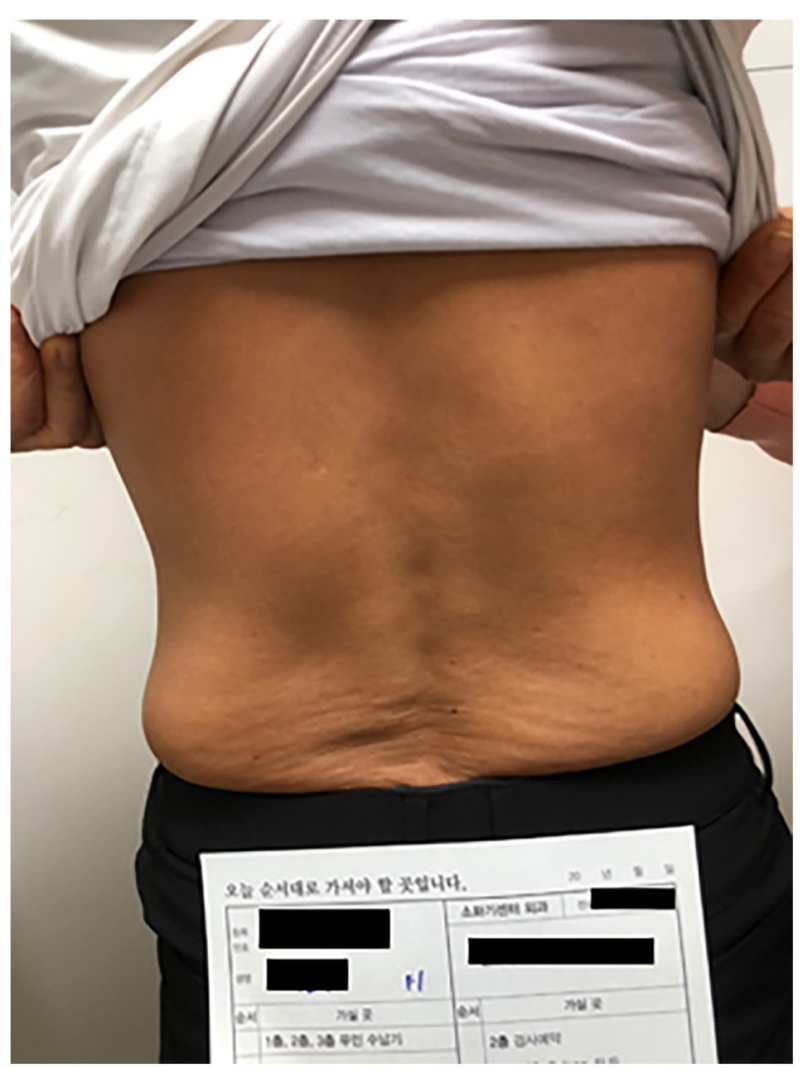

Figure 8. Patient's tanned skin due to extensive sunlight exposure of the torso and limbs.

1 h per day (Fig. 8). Her vitamin D level was higher than the of normal range at the time of the CR, although we did not record her initial vitamin $\mathrm{D}$ level. The patient took no 
vitamin D supplements or injections; therefore we infer that her high vitamin D level was due solely to the direct absorption of sunlight. Recent studies investigating the relationship between vitamin $\mathrm{D}$ and cytotoxic $\mathrm{T}$ cells that attack cancer suggested that an increased vitamin $\mathrm{D}$ level may promote $\mathrm{CD}^{+} \mathrm{T}$ cell tumor infiltration, which would result in a lower tumor burden. Karkeni et al (20) have reported that vitamin D supplements contributed to significant increases in $\mathrm{CD}^{+} \mathrm{T}$ cell recruitment to the tumor site and $\mathrm{CD}^{+} \mathrm{T}$ cell activation compared with the control. We cautiously infer that vitamin D synthesized by sunshine may have played a role in activating cytotoxic $\mathrm{T}$ cells, which contributed to the patient's CR. This theory should be explored in both laboratory and clinical trials.

Our patient had a right-sided tumor harboring wild-type RAS. In their CORRELATE study, Ducreux et al (21) showed similar OS and PFS after regorafenib treatment between leftand right-sided mCRC patients; however, Yoon et al (9) found that left-sided primary tumors were associated with improved outcomes, including PFS, after treatment in their multivariate analysis (2.6 vs. 1.9 months, $\mathrm{P}=0.04$ ). The role of the RAS mutation status remains controversial; in the CORRECT study, no relationship was detected between the KRAS mutation status and outcomes following regorafenib treatment, whereas in the REBECCA study, mutations were found to be a prognostic factor for poor survival $(2,22)$. Further studies are essential to confirm whether these factors influence the outcomes of regorafenib treatment.

As suggested by Yoshino et al (4), we stopped regorafenib treatment after the 17th cycle due to the lack of remnant tumor evident on both CT and PET-CT. Remarkably, the patient has not experienced recurrence 2 years since treatment cessation; no similar findings have been reported previously. Although the regorafenib dose had to be reduced due to palmar-plantar erythrodysesthesia syndrome, there were no other severe side effects such as liver function deterioration or hypertension. Notably, the dose reduction did not appear to diminish the antitumor effect of regorafenib; however, further studies are needed for confirmation.

In summary, this case study suggests that the high mutational burden carried by MSI-H tumor acts as a neoantigen in the innate immune system, and that regorafenib plays an additional role in enhancing tumor antigen recognition by immune cells. An increased vitamin D level via extensive sunlight exposure may also have encouraged cytotoxic T cell recruitment and activation to the tumor microenvironment. All of these factors appear to have contributed to the patient's CR. However, well-designed preclinical and clinical studies are required to demonstrate the mechanism behind this response.

In conclusion, this case report presents the first patient with MSI-H mCRC to achieve a CR to regorafenib treatment after failure of conventional palliative chemotherapy. The mechanism behind this response requires further evaluation in larger studies, to identify which mCRC patients are most likely to benefit from regorafenib.

\section{Acknowledgements}

Not applicable.

\section{Funding}

The present study was supported by the 2015 Inje University Busan Paik Hospital Research Grant (Busan Paik-2015).

\section{Availability of data and materials}

The datasets used and/or analyzed during the current study are available from the corresponding author on reasonable request.

\section{Authors' contributions}

$\mathrm{HB}$ was involved in manuscript writing and acquisition of data. HJL wrote the manuscript. JuP designed the study and gathered patient information. HYP was involved in pathology review and case review. JiP was involved in image (CT) review. SL was involved in image (PET) review. KBB was involved in conception of the case report and analysis of the case. $\mathrm{HB}$ and $\mathrm{KBB}$ confirm the authenticity of all the raw data. HB, HJL and $\mathrm{KBB}$ have reviewed all the raw data separately, and have confirmed that the results are correctly described. All authors read and approved the final manuscript.

\section{Ethics approval and consent to participate}

This type of study does not require ethics approval due to its retrospective nature. The patient consented to the treatment.

\section{Patient consent for publication}

Informed oral consent was obtained from the patient.

\section{Competing interests}

The authors declare that they have no competing interests.

\section{References}

1. Wilhelm SM, Dumas J, Adnane L, Lynch M, Carter CA, Schütz G, Thierauch KH and Zopf D: Regorafenib (BAY 73-4506): A new oral multikinase inhibitor of angiogenic, stromal and oncogenic receptor tyrosine kinases with potent preclinical antitumor activity. Int J Cancer 129: 245-255, 2011.

2. Grothey A, Van Cutsem E, Sobrero A, Siena S, Falcone A, Ychou M, Humblet Y, Bouché O, Mineur L, Barone C, et al: Regorafenib monotherapy for previously treated metastatic colorectal cancer (CORRECT): An international, multicentre, randomised, placebo-controlled, phase 3 trial. Lancet 381: 303-312, 2013.

3. Li J, Qin S, Xu R, Yau TC, Ma B, Pan H, Xu J, Bai Y, Chi Y, Wang L, et al: Regorafenib plus best supportive care versus placebo plus best supportive care in Asian patients with previously treated metastatic colorectal cancer (CONCUR): A randomised, double-blind, placebo-controlled, phase 3 trial. Lancet Oncol 16: 619-629, 2015.

4. Yoshino K, Manaka D, Kudo R, Kanai S, Mitsuoka E, Kanto S, Hamasu S, Konishi S and Nishitai R: Metastatic colorectal cancer responsive to regorafenib for 2 years: A case report. J Med Case Rep 11: 227, 2017.

5. U.S. Department of Health and Human Services: Common Terminology Criteria for Adverse Events (CTCAE). Version 5.0. U.S. Department of Health and Human Services, Washington, DC, 2017. https://ctep.cancer.gov/protocoldevelopment/electronic_applications/docs/ctcae_v5_quick_reference_5x7.pdf.

6. Eisenhauer EA, Therasse P, Bogaerts J, Schwartz LH, Sargent D, Ford R, Dancey J, Arbuck S, Gwyther S, Mooney M, et al: New response evaluation criteria in solid tumours: Revised RECIST guideline (version 1.1). Eur J Cancer 45: 228-247, 2009. 
7. Kawasaki K, Hamamoto Y, Adachi M, Kanai T and Takaishi $\mathrm{H}$ Early tumor cavitation with regorafenib in metastatic colorectal cancer: A case report. Oncol Lett 11: 231-233, 2016.

8. Moriwaki T, Fukuoka S, Taniguchi H, Takashima A, Kumekawa Y, Kajiwara T, Yamazaki K, Esaki T, Makiyama C, Denda T, et al: Propensity score analysis of regorafenib versus trifluridine/tipiracil in patients with metastatic colorectal cancer refractory to standard chemotherapy (REGOTAS): A Japanese society for cancer of the colon and rectum multicenter observational study. Oncologist 23: 7-15, 2018.

9. Yoon SE, Lee SJ, Lee J, Park SH, Park JO, Lim HY, Kang WK, Park YS and Kim ST: The impact of primary tumor sidedness on the effect of regorafenib in refractory metastatic colorectal cancer. J Cancer 10: 1611-1615, 2019.

10. Schrock AB, Ouyang C, Sandhu J, Sokol E, Jin D, Ross JS, Miller VA, Lim D, Amanam I, Chao J, et al: Tumor mutational burden is predictive of response to immune checkpoint inhibitors in MSI-high metastatic colorectal cancer. Ann Oncol 30: 1096-1103, 2019.

11. Mandal R, Samstein RM, Lee KW, Havel JJ, Wang H, Krishna C, Sabio EY, Makarov V, Kuo F, Blecua P, et al: Genetic diversity of tumors with mismatch repair deficiency influences anti-PD-1 immunotherapy response. Science 364: 485-491, 2019.

12. Innocenti F, Ou FS, Qu X, Zemla TJ, Niedzwiecki D, Tam R, Mahajan S, Goldberg RM, Bertagnolli MM, Blanke CD, et al: Mutational analysis of patients with colorectal cancer in CALGB/SWOG 80405 identifies new roles of microsatellite instability and tumor mutational burden for patient outcome. J Clin Oncol 37: 1217-1227, 2019.

13. André T, Shiu KK, Kim TW, Jensen BV, Jensen LH, Punt C, Smith D, Garcia-Carbonero R, Benavides M, Gibbs P, et al: Pembrolizumab in microsatellite-instability-high advanced colorectal cancer. N Engl J Med 383: 2207-2218, 2020.

14. Grothey A, Prager G and Yoshino T: The mechanism of action of regorafenib in colorectal cancer: A guide for the community physician. Clin Adv Hematol Oncol 12 (Suppl 17): S1-S19, 2019.

15. Arai H, Battaglin F, Wang J, Lo JH, Soni S, Zhang W and Lenz HJ: Molecular insight of regorafenib treatment for colorectal cancer. Cancer Treat Rev 81: 101912, 2019.
16. Abou-Elkacem L, Arns S, Brix G, Gremse F, Zopf D, Kiessling F and Lederle W: Regorafenib inhibits growth, angiogenesis, and metastasis in a highly aggressive, orthotopic colon cancer model. Mol Cancer Ther 12: 1322-1331, 2013.

17. Cannarile MA, Weisser M, Jacob W, Jegg AM, Ries CH and Rüttinger D: Colony-stimulating factor 1 receptor (CSF1R) inhibitors in cancer therapy. J Immunother Cancer 5: 53, 2017.

18. Wu RY, Kong PF, Xia LP, Huang Y, Li ZL, Tang YY, Chen YH, Li X, Senthilkumar R, Zhang HL, et al: Regorafenib promotes antitumor immunity via inhibiting PD-L1 and IDO1 expression in melanoma. Clin Cancer Res 25: 4530-4541, 2019.

19. Fukuoka S, Hara H, Takahashi N, Kojima T, Kawazoe A, Asayama M, Yoshii T, Kotani D, Tamura H, Mikamoto Y, et al: Regorafenib plus nivolumab in patients with advanced gastric or colorectal cancer: An open-label, dose-escalation, and dose-expansion phase Ib trial (REGONIVO, EPOC1603). J Clin Oncol 38: 2053-2061, 2020.

20. Karkeni E, Morin SO, Tayeh BB, Goubard A, Josselin E, Castellano R, Fauriat C, Guittard G, Olive D and Nunès JA: Vitamin D controls tumor growth and $\mathrm{CD}^{+} \mathrm{T}$ cell infiltration in breast cancer. Front Immunol 10: 1307, 2019.

21. Ducreux M, Petersen LN, Öhler L, Bergamo F, Metges JP, de Groot JW, Wang JY, Paredes BC, Dochy E, Fiala-Buskies S, et al: Safety and effectiveness of regorafenib in patients with metastatic colorectal cancer in routine clinical practice in the prospective, observational CORRELATE study. Eur J Cancer 123: 146-154, 2019.

22. Adenis A, de la Fouchardiere C, Paule B, Burtin P, Tougeron D, Wallet J, Dourthe LM, Etienne PL, Mineur L, Clisant S, et al: Survival, safety, and prognostic factors for outcome with regorafenib in patients with metastatic colorectal cancer refractory to standard therapies: Results from a multicenter study (REBECCA) nested within a compassionate use program. BMC Cancer 16: 412, 2016. International (CC BY-NC-ND 4.0) License. 